\title{
Accuracy in the experimental calorimetric study of the crystallization kinetics and predictive transformation diagrams: Application to a Ga-Te amorphous alloy
}

\author{
N. Clavaguera \\ Grup de Física de l'Estat Sòlid, Departament d'Estructura i Constituents de la Matèria, \\ Facultat de Física, Universitat de Barcelona, Diagonal 647, 08028-Barcelona, Spain \\ M. T. Clavaguera-Mora and M. Fontana ${ }^{\text {a) }}$ \\ Grup de Física de Materials I, Departament de Física, Edifici C, Universitat Autònoma de Barcelona, \\ 08193-Bellaterra, Spain
}

(Received 25 March 1996; accepted 12 May 1997)

\begin{abstract}
The uncertainties inherent to experimental differential scanning calorimetric data are evaluated. A new procedure is developed to perform the kinetic analysis of continuous heating calorimetric data when the heat capacity of the sample changes during the crystallization. The accuracy of isothermal calorimetric data is analyzed in terms of the peak-to-peak noise of the calorimetric signal and base line drift typical of differential scanning calorimetry equipment. Their influence in the evaluation of the kinetic parameter is discussed. An empirical construction of the time-temperature and temperature-heating rate transformation diagrams, grounded on the kinetic parameters, is presented. The method is applied to the kinetic study of the primary crystallization of Te in an amorphous alloy of nominal composition $\mathrm{Ga}_{20} \mathrm{Te}_{80}$, obtained by rapid solidification.
\end{abstract}

\section{INTRODUCTION}

Kinetics of phase transformation studies include several experimental techniques; in particular, optical or electron microscopy, $x$-ray or neutron diffraction, electrical resistivity, magnetic susceptibility, Mössbauer spectroscopy, thermal analysis, and dilatometry are currently used. One of the thermal analysis methods, specifically differential scanning calorimetry (DSC), is of particular interest in first order phase transformations. It provides the rate of transformation as a function of time or temperature by measuring the heat released or absorbed during the phase change. Isothermal and continuous heating (or cooling) measurements are readily made, although care must be taken to account for heat capacity changes of the sample during the transformation and the peak-to-peak noise and base line drift in the calorimetric signal. The use of DSC data to evaluate kinetic parameters of solid state reaction is well established (see, for instance, Borchardt and Daniels, ${ }^{1}$ Freeman and Carroll, ${ }^{2}$ Coats \& Redfern, ${ }^{3}$ and Hugo et al. ${ }^{4}$ ).

The goal of kinetic studies is to determine the kinetic parameters which allow extrapolation of the transformation rate, $\dot{x}$, to broader temperature ranges than those accessible by DSC measurement. An important tool in this respect is the use of time-temperature-transformation

\footnotetext{
a)Permanent address: Departamento de Física, Facultad de Ingenieria, Universidad de Buenos Aires, Paseo Colòn 850 (1063), Buenos Aires, Argentina.
}

diagrams. These T-T-T curves are constructed from the explicit dependence of the crystallized fraction, $x$, on annealing temperature and annealing time. Such diagrams plot a series of temperature versus time curves for a fixed value of $x$. Such curves are determined based on crystallization measurements from a series of isothermal measurements. By a different procedure, TemperatureHeating (Cooling) Rate-Transformation or T-HR-T (T-CR-T) curves are constructed as the temperature versus heating (cooling) rate for a fixed value of $x .^{5-9}$

In this paper, the accuracy in the determination of the kinetic parameters for the primary crystallization of an amorphous sample is discussed with reference to $\mathrm{Ga}_{20} \mathrm{Te}_{80}$ alloys, obtained by rapid solidification. The investigation by means of DSC and x-ray diffraction (XRD) allowed characterization of two main crystallization processes. They correspond, respectively, to primary crystallization of Te and secondary crystallization of the high temperature $\mathrm{Ga}_{2} \mathrm{Te}_{5}$ phase. ${ }^{10,11}$ The analysis of the nucleation-growth mechanisms of these processes is still under study.

The experimental details on the calorimetric measurement of the crystallization kinetics are presented in Sec. II. The evaluation of the transformation rate from the experimental data under continuous heating regime is presented in Sec. III. There, a new procedure was developed to perform the kinetic analysis when the heat capacity of the sample changes during the crystallization. The limits in the accuracy in the values obtained for $\dot{x}$ from isothermal DSC data, coming from the peak-to- 
peak noise (often quite substantial with respect to the total calorimetric signal) and from the base line drift were analyzed in Sec. IV. Several procedures exist to evaluate the kinetic parameters from DSC data. ${ }^{1-4,12,13}$ Some of them are used in the present study and the kinetic parameters deduced, with their corresponding errors, are presented in Sec. V. Finally, in Sec. VI a revision of the previously reported empirical method ${ }^{5,7}$ to construct the low-temperature part of both the T-T- $\mathrm{T}^{5}$ and the T-HR- $\mathrm{T}^{7}$ diagrams is presented. The revision is grounded in the need to account for the uncertainties in the crystallization kinetic parameters and data processing when studying complex crystallization behaviors.

\section{EXPERIMENTAL PROCEDURE}

Master alloys of $\mathrm{Ga}-\mathrm{Te}$ with 20 at. \% Ga were prepared by melting mixtures of elemental high quality $(5 \mathrm{~N})$ gallium and tellurium at $600{ }^{\circ} \mathrm{C}$, in graphite crucibles under an $\mathrm{Ar}$ atmosphere. Samples were rapidly quenched under Ar atmosphere to the amorphous state using the piston and anvil technique. ${ }^{14,15}$ Disk-shaped samples with an average thickness of $30 \mu \mathrm{m}$ and about $1 \mathrm{~cm}$ in diameter were studied. The amorphous character of the rapidly quenched samples was verified from the analysis of their XRD patterns using monochromatized $\mathrm{Cu}\left(\mathrm{K}_{\alpha}\right)$ radiation. Their thermal evolution on heating was studied in a Perkin-Elmer DSC-7 differential scanning calorimeter under a dynamic Ar atmosphere. ${ }^{16}$ Continuous heating experiments were performed at scan rates, $\beta$, ranging between 5 and $80 \mathrm{~K} \cdot \mathrm{min}^{-1}$, whereas isothermal experiments were carried out at temperatures, $T$, of 457,460 , and $463 \mathrm{~K}$. The temperature plateau was attained by heating the amorphous samples from room temperature to the annealing temperature at a rate of $80 \mathrm{~K} \cdot \mathrm{min}^{-1}$.

\section{EXPERIMENTAL CALORIMETRIC RESULTS UNDER CONTINUOUS HEATING}

\section{A. Thermal evolution of the amorphous alloy}

Figure 1 shows the DSC curves obtained for the different heating rates used, ranging from 5 to $80 \mathrm{~K} \cdot \mathrm{min}^{-1}$. Three transformations are occurring sequentially with increasing the temperature. The glass transition appears as an endothermic shift in the DSC base line, due to the change of heat capacity in the sample transforming from the amorphous to the undercooled liquid state. Two exothermic transformations follow: the primary crystallization of $\mathrm{Te}$ and the secondary crystallization of $\mathrm{Ga}_{2} \mathrm{Te}_{5} \cdot{ }^{10,11}$ Here, the terms primary and secondary crystallization are used following Ref. 17, since the transforming phase is an undercooled liquid. After the first crystallization process, the base line remains close to the extrapolated base line of the amorphous state, shown as a dashed line in the upper graph of Fig. 1.

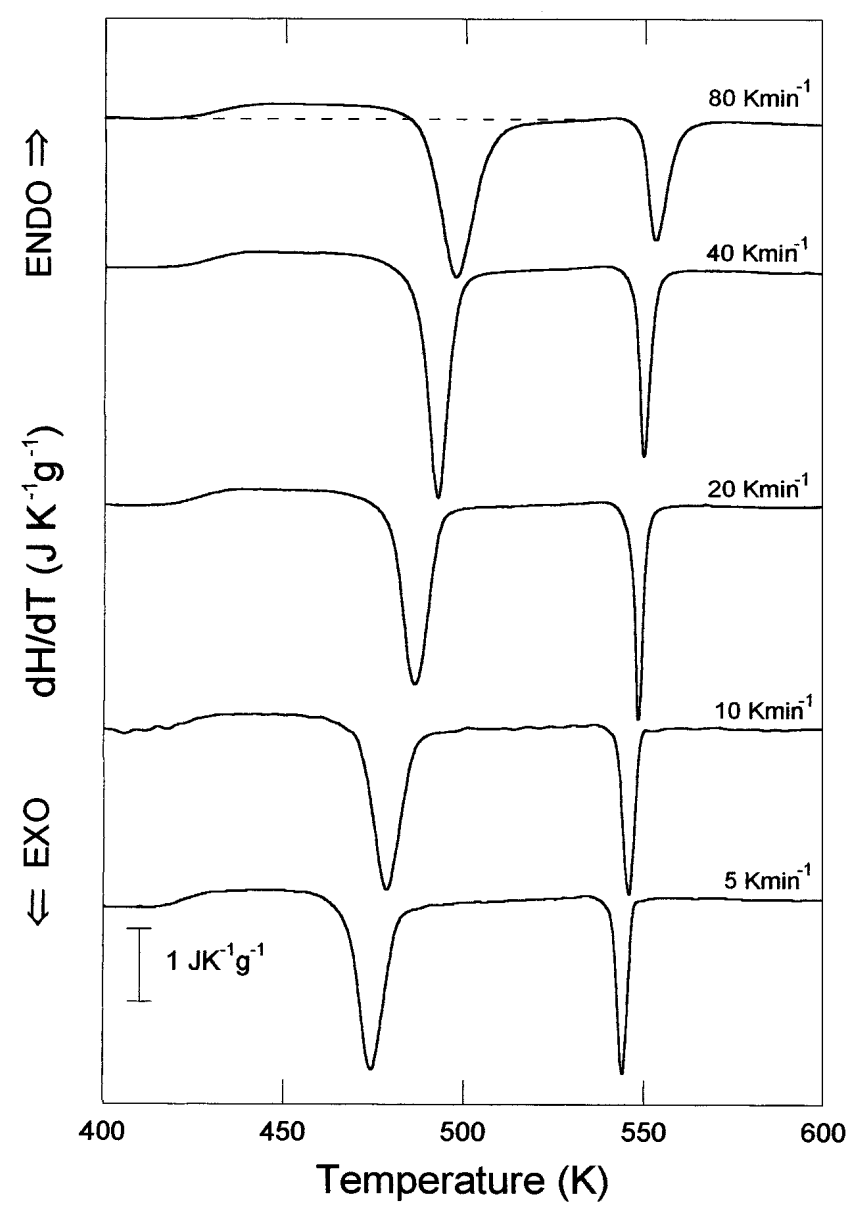

FIG. 1. Apparent heat capacity change obtained on heating the amorphous alloy at different heating rates.

This indicates that there is a significant change of the heat capacity of the sample during its first exothermic transformation. Procedures already exit in the literature to take account of this effect in the overall kinetic analysis of the data. They require estimation of the fraction $x$ transformed from the DSC trace which includes the heat capacity change contribution. ${ }^{18}$ A new procedure is proposed in which the value of $x$ is re-estimated repetitively, by an iterative method, based ultimately on integration of DSC data purged from the change in sample heat capacity contribution.

\section{B. Kinetics of crystallization: Influence of $\Delta C_{p}$}

The experimental data on the kinetics of crystallization allow determination of the evolution of the transformed fraction, $x$, either as a function of temperature, $T$ (under continuous heating or cooling regime), or annealing time, $t$ (under an isothermal regime). Such information is generally obtained from scanning calorimetric experiments by assuming that the instantaneous heat flow evolved from the sample during the transformation, $\dot{Q}$, is proportional to the transformation rate, $\dot{x}$. 
That is,

$$
\dot{Q} \approx \dot{x} \cdot \Delta H,
$$

where $\Delta H$ is the total enthalpy of transformation.

The above expression (1) implicitly assumes a negligible contribution of the change in both (i) composition and (ii) heat capacity of the transforming phase. A more accurate expression of the heat evolved per unit time is ${ }^{18}$

$$
\dot{Q}=\dot{Q}_{\text {reaction alone }}+\dot{Q}_{\Delta C_{p}},
$$

where $\dot{Q}_{\text {reaction alone }}$ is the rate of enthalpy change of the reaction alone and $\dot{Q}_{\Delta C_{p}}$ is the rate of sample heat capacity change from that of the undercooled liquid to that of the crystallization product. This treatment considers that the concept of additive heat flow-as measured by power-compensated DSC - is reasonable. ${ }^{19}$

In eutectic- and polymorphic-type isothermal transformations, the compositions of both the emerging crystalline and the remaining nontransformed phase do not change during the transformation (see appendix). Thus,

$$
\begin{aligned}
\dot{Q}_{\text {reaction alone }} & =\dot{x} \cdot \Delta H, \\
\dot{Q}_{\Delta C_{p}} & =x \cdot \beta \cdot \Delta C_{p},
\end{aligned}
$$

where $\Delta C_{p}$ is the total change in heat capacity of the sample in the transformation. Even when the instantaneous composition of the crystalline nuclei or of the undercooled liquid change during the transformation, as in primary crystallization, Eqs. (3) and (4) are used, as a good approximation (see appendix). Obviously, Eqs. (2) to (4) reduce to Eq. (1) under isothermal regime $(\beta=0)$ or even under continuous heating in the particular case where $\Delta C_{p}$ is so small that the second term in Eq. (2) is negligible.

Under continuous heating at a rate $\beta$, the value of $\Delta C_{p}$ is directly obtained from the shift of the base line of the DSC curve, between the exotherm onset and the exotherm end (see Fig. 3). Let us consider that the exotherm onset occurs at the temperature $T_{\text {on }}$ (the highest temperature before the transformation for which $x=0$ and $\dot{x}=0$ ). Similarly, the exotherm end occurs at $T_{\text {end }}$ and then $\dot{x}$ becomes zero again with $x=1$. One has to obtain the actual value of $\Delta H$, and, for each temperature $T$, in the range $T_{\text {on }} \geqslant T \geqslant \mathrm{~T}_{\text {end }}$, the actual values of $x$ and $\dot{x}$. They come from the experimental knowledge of $\dot{Q}$, and the values of $\beta$ and $\Delta C_{p}$. The procedure presented here is an iterative method where the fraction transformed is re-estimated at every step of the iteration process and purged sequentially from the heat capacity change contribution term.

The iteration procedure starts with instantaneous values of $x$, named $x_{0}$, calculated assuming for $\dot{Q}_{\Delta C_{p}}$ a linear shape between the onset and the end of the transformation exotherm. An example of the way used

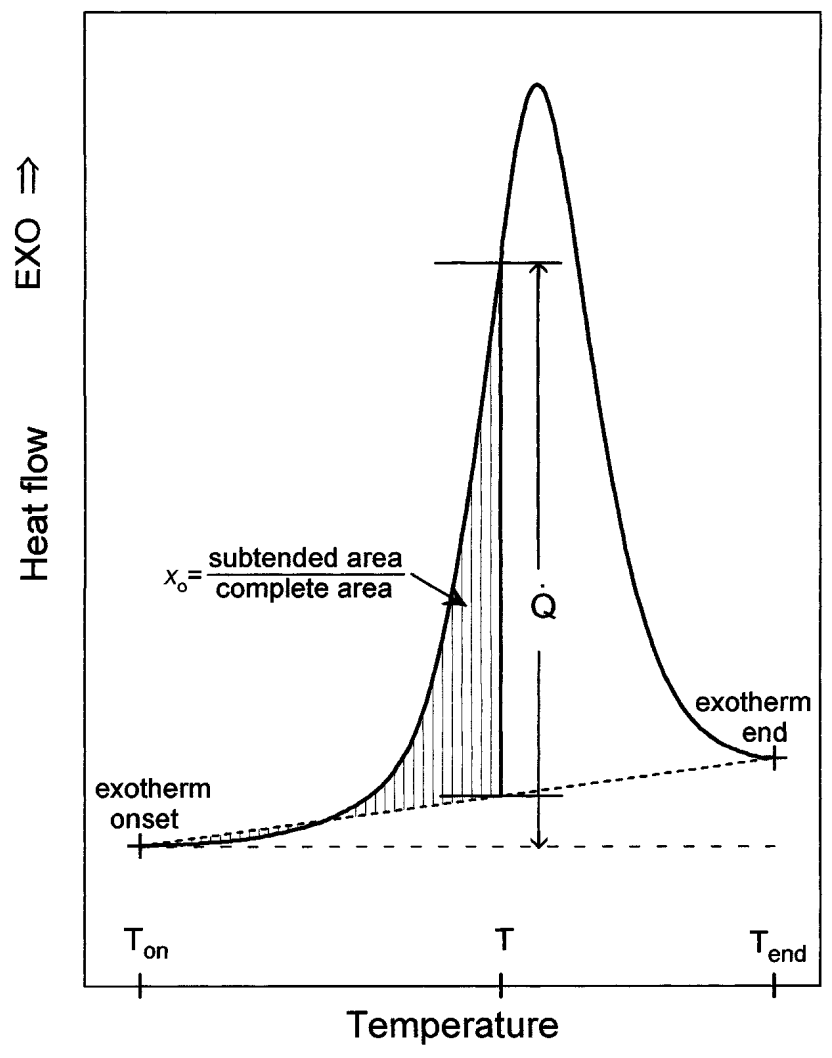

FIG. 2. Diagram of a DSC curve including a crystallization peak and a heat capacity change, indicating the subtended area and the evaluation of $x_{0}$ for a particular temperature.

to obtain the $x_{0}$ values is shown in Fig. 2. For increasing values of the iteration step, $i>0$, the values of $\Delta H_{i}$, $\dot{x}_{i}$ and $x_{i}$ are re-estimated, by use of the following equations:

$$
\begin{aligned}
\Delta H_{i} & =\frac{1}{\beta} \int_{T_{\mathrm{on}}}^{T_{\mathrm{end}}}\left(\dot{Q}-x_{i-1} \cdot \beta \cdot \Delta C_{p}\right) d T, \\
\dot{x}_{i} & =\left(\dot{Q}-x_{i-1} \beta \Delta C_{p}\right) / \Delta H_{i}, \\
x_{i} & =\frac{1}{\beta} \int_{T_{\mathrm{on}}}^{T} \dot{x}_{i} d T .
\end{aligned}
$$

Equation (5) gives the estimated value of the crystallization enthalpy. Equation (6) gives the ratio $\left(\dot{x}_{i}\right)$ between the estimated heat flow due to the reaction alone and the enthalpy of crystallization. Equation (7) evaluates the instantaneous value of the fraction transformed, $x_{i}$, by integration of $\dot{x}_{i}$.

The outlined procedure creates data sets whose proximity to the actual data sets is performed by selecting a value $\epsilon$ for which the iteration stops at a step $k$ when

$$
\left|\Delta H_{k}-\Delta H_{k-1}\right| \leqslant \epsilon \cdot \Delta H_{k} .
$$

This means that the added enthalpy at step $k$ is negligible. 
The results obtained by the above procedure are illustrated in Fig. 3 that shows the original DSC experimental results for the first crystallization exotherm of the $20 \mathrm{~K} \cdot \mathrm{min}^{-1}$ curve (thick line), split into $\dot{Q}_{\text {reaction alone }}$ and $\dot{Q}_{\Delta C_{p}}$ (thin lines). In the present application the selected value was $\epsilon=1 \times 10^{-4}$ and iteration stopped when $k=8$. The approximate heat capacity term obtained with a single iteration is also shown in this figure, as a dashed line. Its shape is very close to the one obtained after several iterations and may be compared to the result of the method already described in Ref. 18 .

\section{EXPERIMENTAL CALORIMETRIC RESULTS UNDER AN ISOTHERMAL REGIME: EVALUATION OF EXPERIMENTAL ERRORS}

As very sensitive DSC equipment have become available, they are widely used as a tool to study the kinetics of transformations. Nevertheless, there are limits to the accuracy in the determination of the rate of reaction, the most important of which in crystallization studies is the uncertainty of the base line position. ${ }^{16} \mathrm{We}$ have already pointed out the importance of the role of the variation of the heat capacity inherent to the change of the sample from the disordered to the crystalline

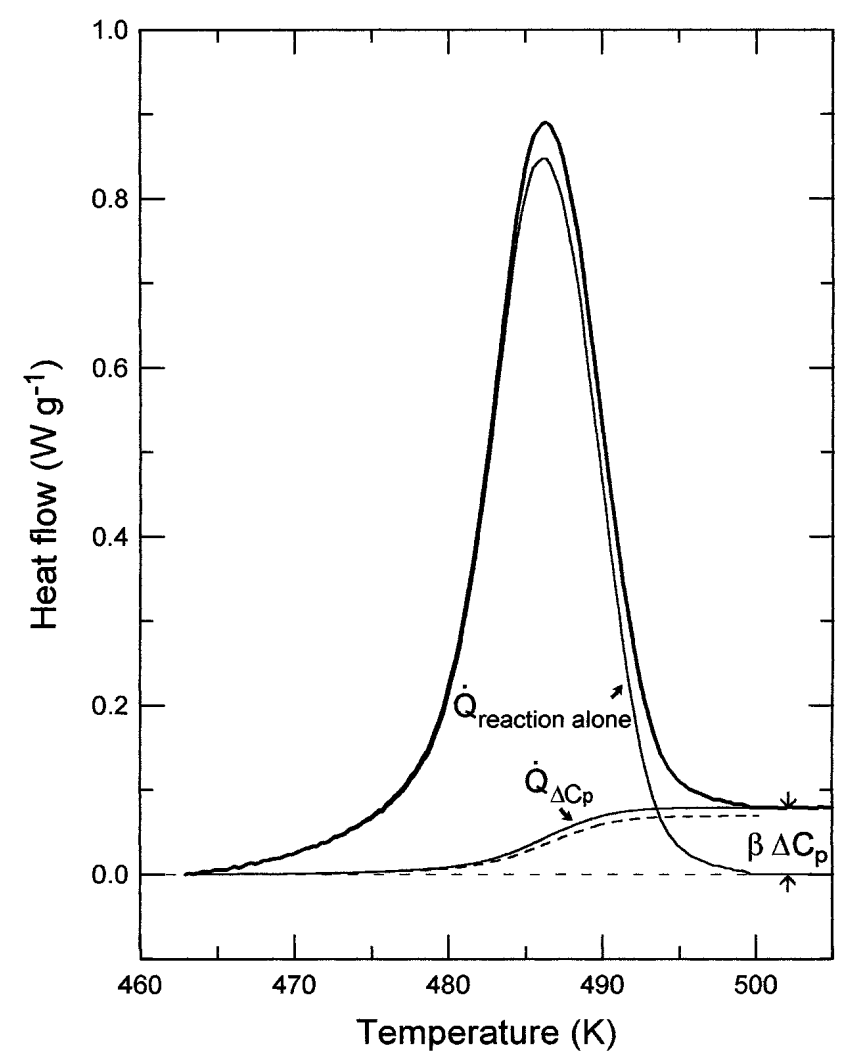

FIG. 3. Partition of the overall DSC curve (thick lines) in one part,

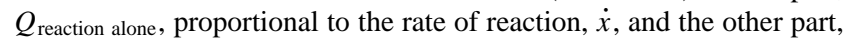
$Q_{\Delta C_{p}}$, proportional to the crystallized fraction, $x$. Approximate heat capacity term (dashed line) with a single iteration. state. Other sources of error come from instrumentally based base line instability during the experiment, and the uncertainty associated with the determination of the exact positions of the onset, and the end of a transformation exotherm (or any other thermal event) in the DSC trace. Both effects are especially important under isothermal conditions and we will treat them together, since they may occur simultaneously.

The following illustration is for the crystallization exotherm obtained at $457 \mathrm{~K}$ (see Fig. 4) under the following assumptions:

\section{Base line instability}

$\pm 10 \mu \mathrm{W}$ rms peak-to-peak noise

$\pm 10 \mu \mathrm{W}$ base line drift in $30 \mathrm{~min}$ isothermal measuring time

Uncertainty in time

$\pm 20 \mathrm{~s}$ for the onset and end time.

The calculated values of the relative errors in both the reaction rate, $|\Delta \dot{x}| / \dot{x}$, and the transformed fraction, $|\Delta x| / x$, are plotted, respectively, in Figs. 5 and 6 as a function of $x$. As expected, the relative error on the reaction rate, Fig. 5, is quite high at the onset and end of the exotherm but it remains lower than $4 \%$ in most of the plotted range. With regard to the crystallized fraction, Fig. 6 illustrates how the relative error decreases toward a value of about $4 \%$ as the transformation proceeds. However, in the early stages of transformation it exceeds $10 \%$. The curve presented in Fig. 5 is not symmetric with respect to the value $x=0.5$ because of the shape of the

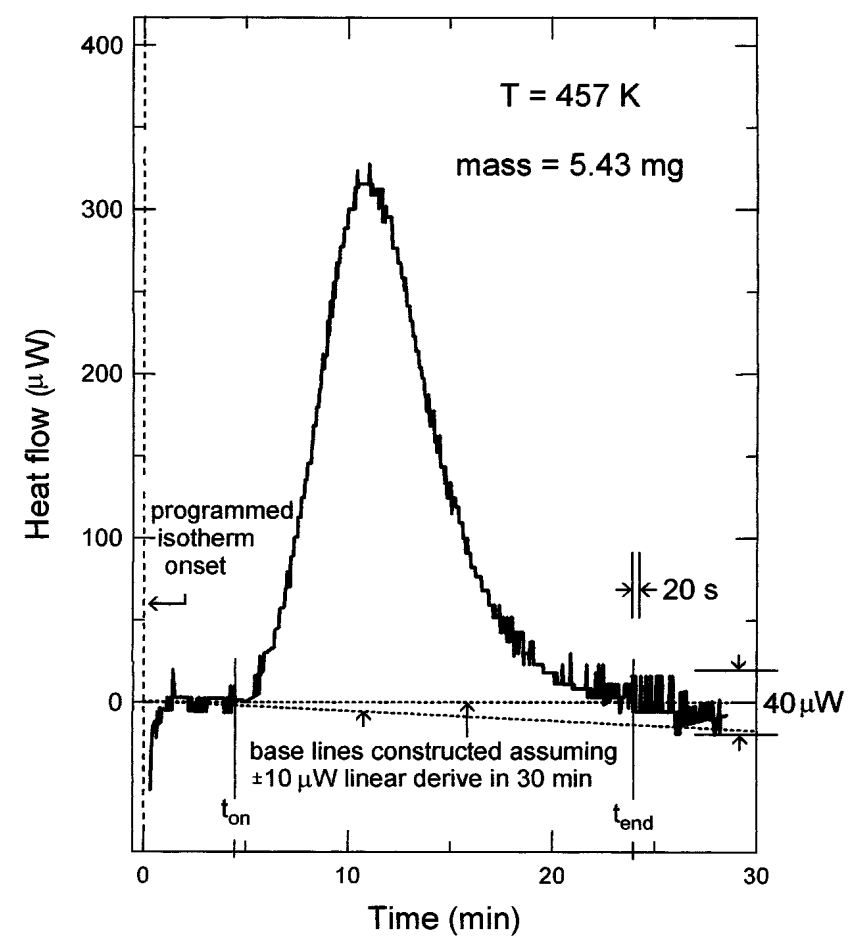

FIG. 4. Isothermal DSC transformation peak at $457 \mathrm{~K}$. 


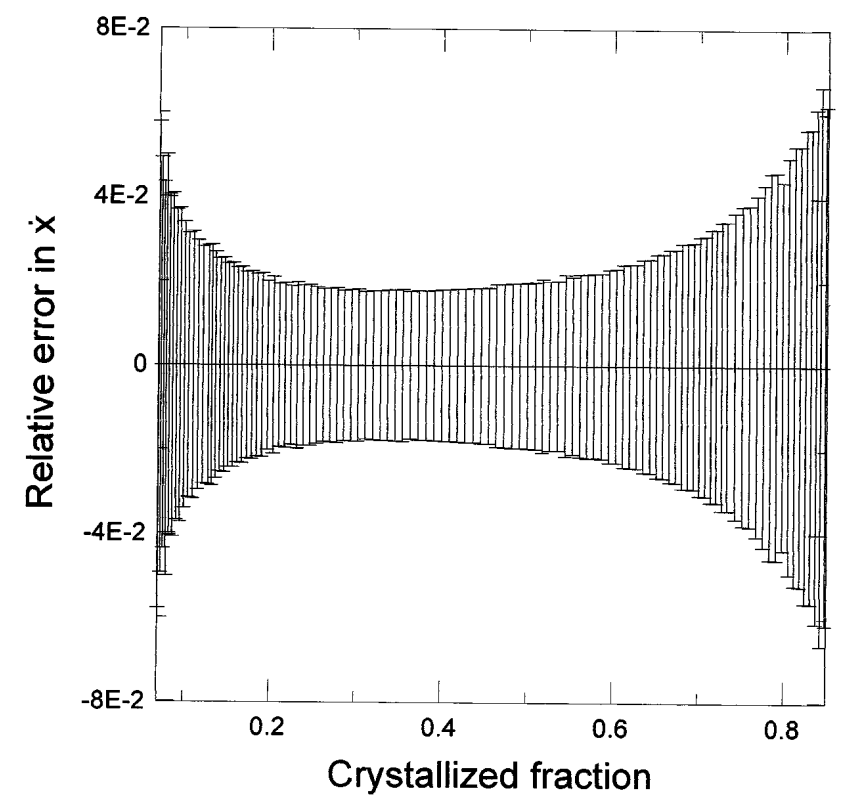

FIG. 5. Relative error in the rate of crystallization, $\pm|\Delta \dot{x} / \dot{x}|$ (shown with bars), versus the crystallized fraction.

isothermal transformation exotherm used to analyze the errors. That means that, in general, the relative errors will vary with the form of the exotherm. However, the results presented here are good estimates of the expected uncertainty in kinetic analysis by DSC. One has to have in mind this fact in the analysis of the data. For instance, in the so-called Avrami method ${ }^{20,21}$ to obtain kinetic exponents one calculates the value of $\ln [-\ln (1-x)]$ versus $\ln (t)$. Then for the time at which one considers that $x \leqslant 0.05$ one has $|\Delta x| / x \geqslant \pm 0.1$ and, consequently one may expect $|\Delta \ln [-\ln (1-x)]| \geqslant \pm 0.2$.

\section{DETERMINATION OF THE KINETIC PARAMETERS: APPARENT ACTIVATION ENERGY AND PRE-EXPONENTIAL FACTOR}

Figures 7 and 8 show, respectively the kinetic data obtained for the first crystallization process under continuous heating $\dot{x}=\dot{x}(T, \beta)$ and isothermal $\dot{x}=\dot{x}$ $(t, T)$ regimes. We will assume that the rate of reaction, $\dot{x}$, may be written as

$$
\dot{x}=K(T) \cdot f(x),
$$

where the function $f(x)$ reflects the mechanisms driving the crystallization and the rate constant follows the Arrhenius law, that is

$$
K(T)=K_{0} \exp (-E / k T),
$$

with $K_{0}$ the pre-exponential factor and $E$ the apparent activation energy of the rate constant $K(T)$.

Our goal is the evaluation of $E, K_{0}$, and $f(x)$ from the results plotted in Figs. 7 and 8. The value of $E$ is

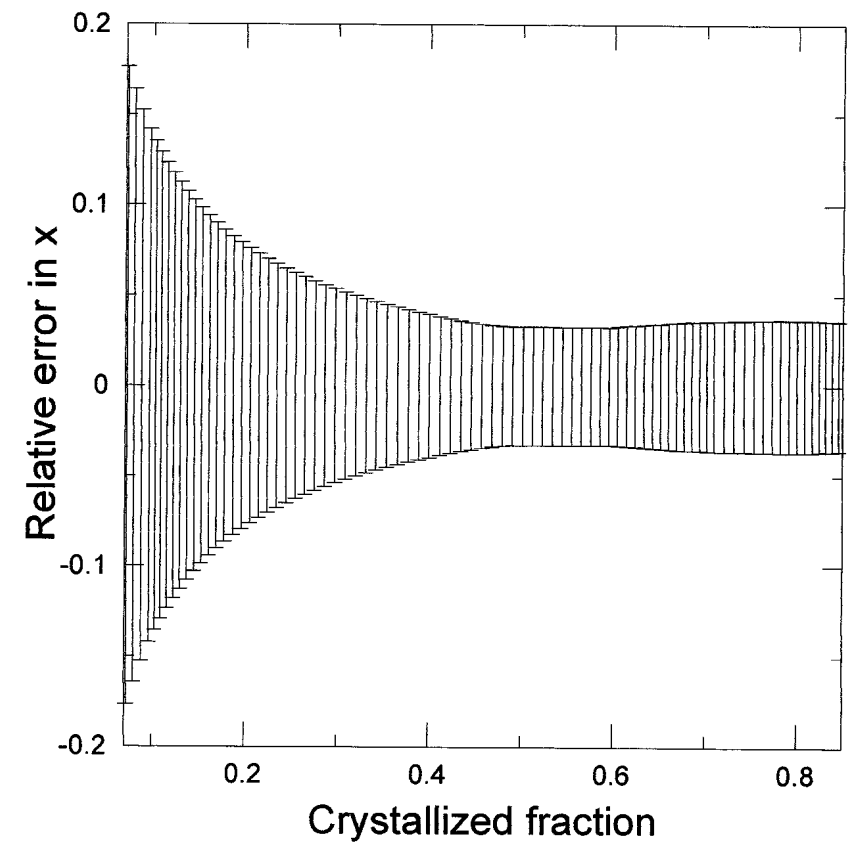

FIG. 6. Relative error in the crystallized fraction, $\pm|\Delta x / x|$ (shown with bars), versus $x$.

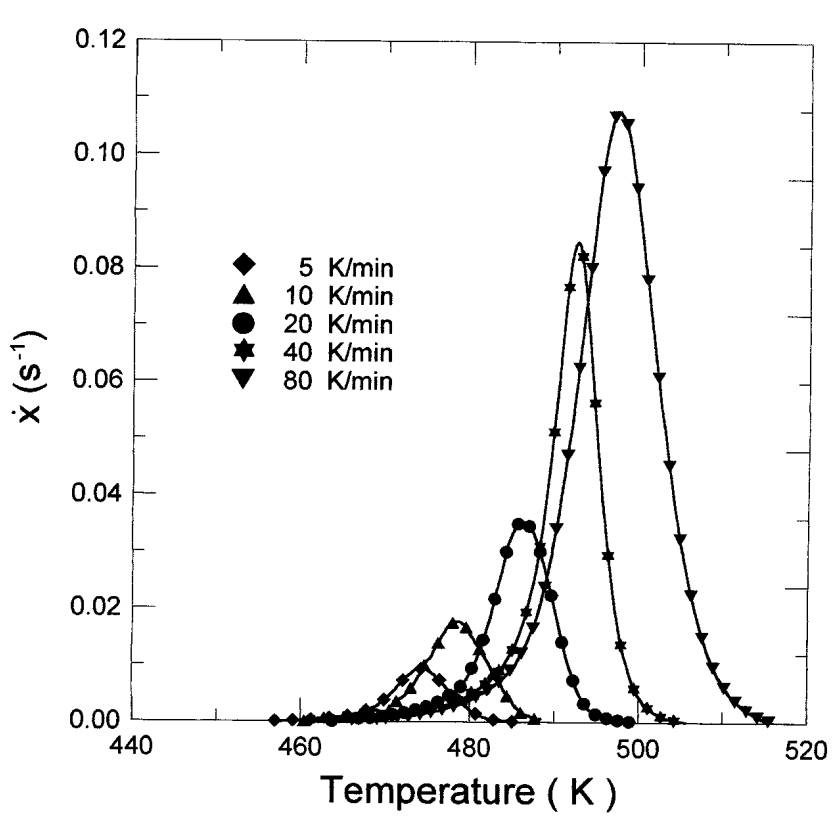

FIG. 7. Rate of crystallization versus temperature obtained on heating the amorphous alloy at scan rates in between 5 and $80 \mathrm{~K} \cdot \mathrm{min}^{-1}$.

often deduced by the so-called "exotherm peak" methods which consider the condition fulfilled at the maximum rate of reaction. In continuous heating regime the most used $^{21}$ are the Kissinger ${ }^{12}$ and Ozawa ${ }^{13}$ methods and in an isothermal regime the time at maximum reaction rate method. ${ }^{22}$ These methods provide the value of $E$ without any need to evaluate $\dot{x}$ and $x$. The values obtained from the Kissinger and time at maximum 


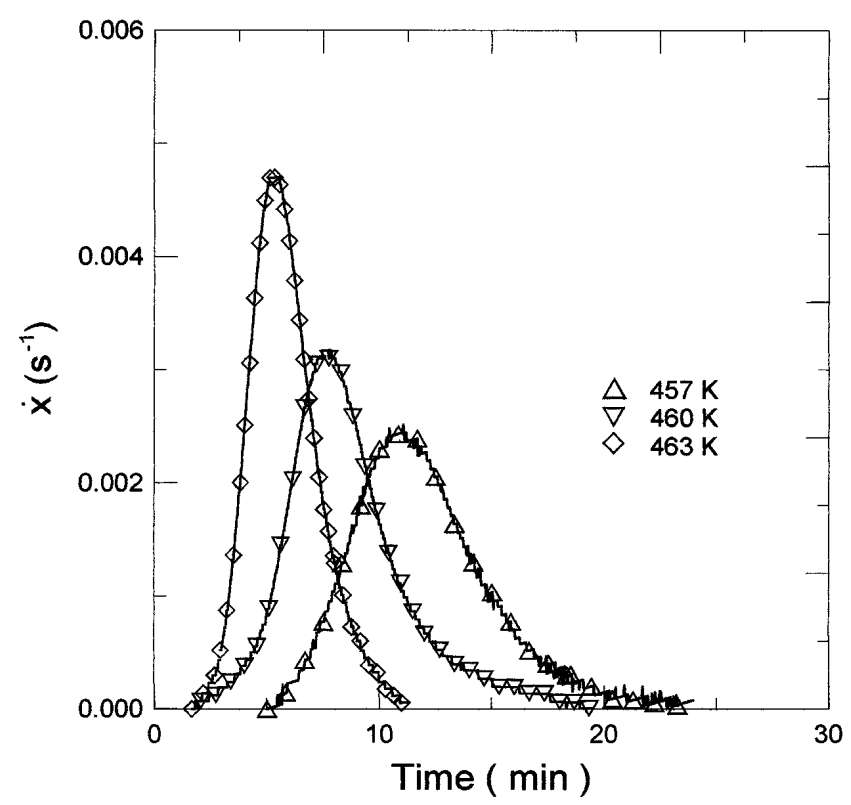

FIG. 8. Rate of crystallization versus time obtained under isothermal annealing at several temperatures.

reaction rate methods are quoted in Table $\mathrm{I}$, whereas the corresponding plots are shown in Fig. 9.

Equations (9) and (10) may be rewritten in the form

$$
\dot{x}=\left\{K_{0} \cdot f(x)\right\} \exp (-E / k T) .
$$

In the so-called the multiple scans method, ${ }^{21}$ the simultaneous evaluation of $E$ and $K_{0} f(x)$ is performed from the Arrhenius plot of Eq. (11) at fixed values of $x$, either from continuous heating or from isothermal data. Figure 10 shows the multiple scan analysis performed for several values of $x$. Full lines correspond to the least squares fitting of each particular set of data (isothermal or continuous heating) for the $x$ values stated in the legend. Both the activation energy (slope of the thick lines) and the $K_{0} f(x)$ values ( $y$-intercept, in exponential form) obtained from the Arrhenius plot shown in Fig. 10, are quoted in Table I.

In the multiple scan method, any inaccuracy in the value obtained for $E,|\Delta E|$, rebounds on the error in evaluating $\ln \left\{K_{0} f(x)\right\},\left|\Delta \ln \left\{K_{0} f(x)\right\}\right|$. Assuming $T$ and $x$ were free of error, it results in:

$$
\left|\Delta \ln \left\{K_{0} f(x)\right\}\right|=|\Delta E| / k T .
$$

For example, different values for $E$ are quoted in Table I in the multiple scans method, for a fixed value of $x=$ 0.55 , namely $E=1.54 \mathrm{eV}$ (from isothermal data) and $E=2.05 \mathrm{eV}$ (from continuous heating data). Taking a mean value of $10^{3} / T \approx 2.1 \mathrm{~K}^{-1}$, and considering $|\Delta E|= \pm 0.26 \mathrm{eV}$, Eq. (9) gives $\left|\Delta \ln K_{0} \cdot f(x)\right| \approx \pm 7$, i.e., about three orders of magnitude uncertainty in the evaluation of $K_{0} \cdot f(x)$. Consequently, different values for $K_{0} \cdot f(x)$ may be obtained from quite similar values of the apparent activation energy. The more accurate values of the transformation rate are often obtained for its maximum value, i.e., close to the exotherm maximum. This is one of the major reasons for which methods, such as Kissinger or isothermal time at maximum rate methods, give quite good estimates of $E$.

The uncertainty in the values of $\dot{x}$ and $T$ is included by symbols with error bars in Fig. 10. They also limit the accuracy in the determination of $E$ and $K_{0} \cdot f(x)$. Even if there is a large scatter, the values reported in Table I suggest that in the limited temperature interval scanned experimentally $(460-500 \mathrm{~K})$ the kinetics of crystallization may be considered to agree with the assumption of a constant activation energy. Therefore, it is assumed in the following that a unique set of empirical kinetic parameters reproduces the experimental data for both isothermal and continuous heating regimes in the primary crystallization of Te analyzed. This set is reported in Table I and drawn (dashed lines) in Fig. 10.

\begin{tabular}{|c|c|c|c|c|}
\hline & \multicolumn{2}{|c|}{ Kissinger method } & \multicolumn{2}{|c|}{ Isothermal time data } \\
\hline \multirow[t]{2}{*}{$E(\mathrm{eV})$} & \multicolumn{2}{|c|}{$2.08 \pm 0.2$} & \multicolumn{2}{|c|}{$2.13 \pm 0.2$} \\
\hline & \multicolumn{4}{|c|}{ Multiple scans method } \\
\hline Isothermal data & $x=0.1$ & 0.3 & 0.55 & 0.7 \\
\hline$E(\mathrm{eV})$ & $1.75 \pm 0.2$ & $1.73 \pm 0.2$ & $1.54 \pm 0.2$ & $1.82 \pm 0.2$ \\
\hline$K_{0} f(x)\left(10^{17} \mathrm{~s}\right)$ & $0.22 \pm 0.1$ & $0.25 \pm 0.08$ & $0.002 \pm 0.001$ & $2.3 \pm 0.8$ \\
\hline Continuous heating data & $x=0.1$ & 0.3 & 0.55 & 0.7 \\
\hline$E(\mathrm{eV})$ & $1.95 \pm 0.2$ & $2.04 \pm 0.2$ & $2.05 \pm 0.2$ & $2.06 \pm 0.2$ \\
\hline \multirow[t]{2}{*}{$K_{0} f(x)\left(10^{19} \mathrm{~s}\right)$} & $0.26 \pm 0.1$ & $5.1 \pm 2$ & $4.7 \pm 1$ & $2.2 \pm 1$ \\
\hline & \multicolumn{4}{|c|}{ Selected set of kinetic parameters } \\
\hline Crystallized fraction & $x=0.1$ & 0.3 & 0.55 & 0.7 \\
\hline$K_{0} f(x)\left(10^{19} \mathrm{~s}\right)$ with $E=(2.08 \pm 0.2) \mathrm{eV}$ & $8.26 \pm 0.4$ & $18.2 \pm 0.9$ & \pm 0.9 & $12.8 \pm 0.6$ \\
\hline
\end{tabular}

TABLE I. Crystallization parameters evaluated by DSC. 


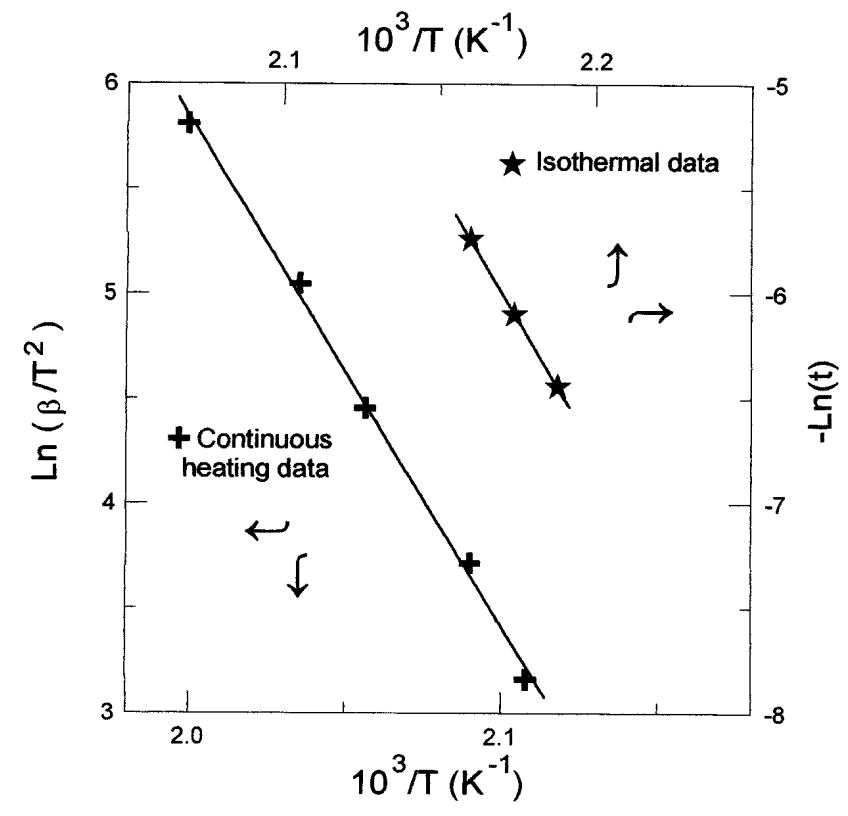

FIG. 9. Plot of both $\operatorname{Ln}\left(\beta / T^{2}\right)$ - with $\beta T^{2}$ in units of $\mathrm{K}^{-1} \min ^{-1}-$ $T$ being the peak temperature obtained under continuous heating at scan rates in the range $5-80 \mathrm{~K} \cdot \min ^{-1}$ and $\operatorname{Ln}(t)-t$ being the peak time (in $s$ ) - obtained under isothermal annealing versus inverse of temperature.

\section{EMPIRICAL CONSTRUCTION OF THE T-T-T AND T-HR-T DIAGRAMS}

As recently derived, ${ }^{5-9}$ the transformation diagrams that describe nonequilibrium crystallization under isothermal or continuous heating regimes, namely the T-T-T (Time-Temperature-Transformation) and T-HR-T (Temperature-Heating Rate-Transformation) diagrams, may be empirically constructed, at least in a limited temperature range, by assuming a specific form of the rate of reaction. The low temperature part of these curves is obtained from the hypothesis of an apparent activation energy.

The empirical construction of the T-T-T and T-HR$\mathrm{T}$ diagrams is grounded on the recognition that Eq. (11) gives, in differential form, either the function $x=x(t)$ at a given $T$ or the function $x=x(T)$ at a given $\beta$, when applied to isothermal and continuous change of temperature regimes, respectively. ${ }^{5,7}$ Namely, under isothermal conditions (at temperature $T$ ), the integrated form of Eq. (11) is

$$
g(x) / K_{0}=t \exp (-E / k T),
$$

where $g(x)$ is defined as

$$
g(x) / K_{0} \equiv \int_{0}^{x} \frac{d x}{K_{0} f(x)}
$$

Equation (13) can be written the form $x=x(T), t$. This equation, when represented as the temperature versus time needed to crystallize a fixed fraction $x$ of the

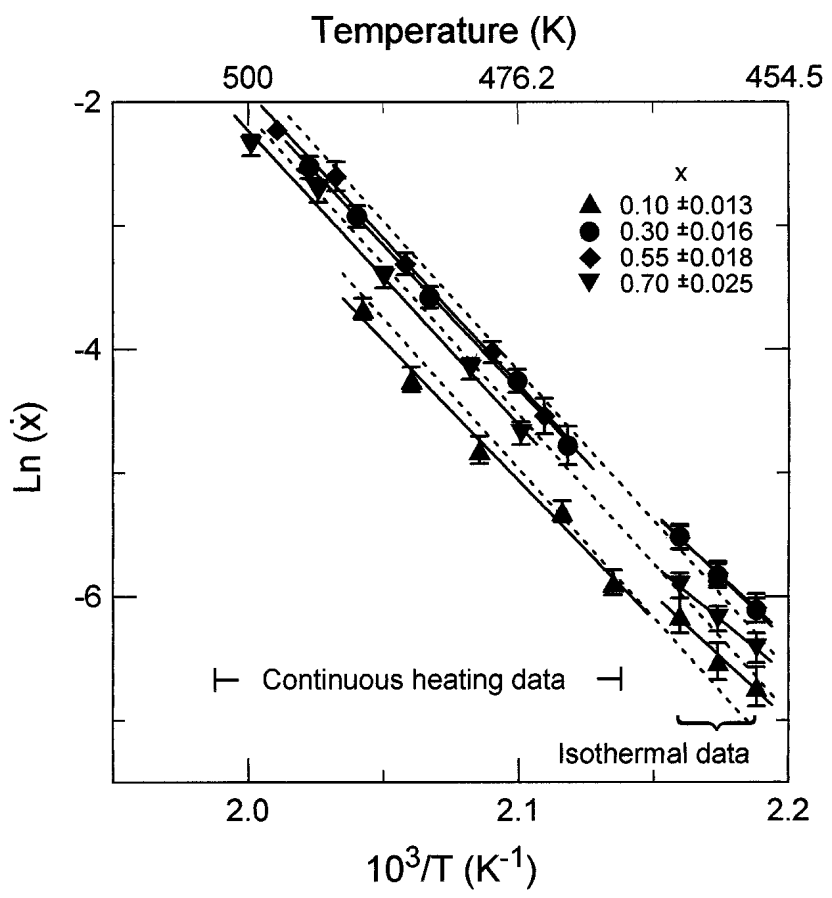

FIG. 10. Plot of $\operatorname{Ln}(\dot{x})$ - with $\dot{x}$ in units of $\mathrm{s}^{-1}$ - versus inverse of temperature for a fixed value of the crystallized fraction. Full lines correspond to the least square fitting of each particular set for a fixed value of $x$ while the dashed line is drawn for the mean value of the activation energy.

material gives a particular T-T-T curve in the T-T-T diagram.

By a similar procedure, integration of Eq. (11) under a constant scan rate $\beta$ gives

$$
g(x) / K_{0}=\frac{1}{\beta} \int_{0}^{T} \exp (-E / k T) d t,
$$

with the same definition, Eq. (14), of $g(x) / K_{0}$. Here, the integration may be carried out within the limits given by the duration of the process, namely from $T_{\text {on }}(x=0)$ to $T(0<x<1)$. In other words, the contribution of the integration between $0<T<T_{\text {on }}$ is negligible. The extent of crystallization is a function only of temperature and scan rate, i.e., $x=x(T, \beta)$. Assuming a fixed value of $x$, the plot of the temperature against the heating rate produces a specific T-HR-T curve in the T-HR-T diagram.

The main difficulty in the above presentation is the evaluation of $g(x) / K_{0}$ by Eq. (13). The instantaneous value of the product $K_{0} f(x)$ is evaluated from the experimental $\dot{x}$ data by introducing the apparent activation energy, in Eq. (11), rewritten in the form

$$
K_{0} f(x)=\dot{x} \cdot \exp (E / k T) .
$$

As already mentioned, the integration may be carried out within the limits given by the duration of the process, namely from $x=\dot{x}=0\left(T_{\text {on }}\right)$ to $x(T)$. However, any 
uncertainty in the values of $\dot{x}$ at the exotherm onset may produce an integrand that diverges when $T \rightarrow T_{\text {on }}$ (since both $\dot{x}$ and $K_{0} f(x) \rightarrow 0$ ).

Since both the T-T-T and T-HR-T transformation diagrams plot a limited series of $n$ curves, each one for a fixed value of the transformed fraction, $x_{a}$ (with $a=1, \ldots, n)$, their empirical construction may be performed without the use of Eq. (13) to obtain $g\left(x_{a}\right) / K_{0}$. The proposed methodology for getting the T-HR-T diagram is the following one. For a series of fixed values of the transformed fraction, $x_{a}(a=1, \ldots, n)$, let us consider the temperatures, $T_{a o}$, at which $x_{a}$ is attained when scanning at a particular value of the heating rate, $\beta_{o}$. Let us name $\beta_{a}$ any other value of the heating rate for which the same value of the transformed fraction, $x_{a}$, will be attained at a temperature $T_{a}$. For any of the couples $\left(T_{a}, \beta_{a}\right)$ the following relationship is satisfied:

$$
\begin{aligned}
g\left(x_{a}\right) / K_{0} & =\frac{1}{\beta_{o}} \int_{0}^{T_{a o}} \exp (-E / k T) d t \\
& =\frac{1}{\beta_{a}} \int_{0}^{T a} \exp (-E / k T) d T .
\end{aligned}
$$

This equation allows us to obtain $T=T(\beta)$ for $x=x_{a}$, that is a T-HR-T curve, by the simple evaluation of the integral of $\exp (-E / k T)$.

Very similarly, from a previous knowledge of $g\left(x_{a}\right) / K_{0}$ deduced from continuous heating data by Eq. (17), the time duration, $t_{a}$, needed to achieve a transformed fraction $x_{a}$, at a temperature $T_{a}$, satisfies the equation

$$
g\left(x_{a}\right) / K_{0}=t_{a} \exp \left(-E / k T_{a}\right) .
$$

These data may be plotted in the form $T=T(t)$ for $x=$ $x_{a}$ as one of the T-T-T curves in the T-T-T diagram.

The low temperature part of the T-T-T and T-HR-T curves is constructed with the above-mentioned procedure using the selected value of the apparent activation energy quoted in Table I, and the continuous heating data at $\beta_{o}=20 \mathrm{~K} \cdot \mathrm{min}^{-1}$. The respective T-T-T and T-HR-T transformation diagrams are shown in Figs. 11 and 12. Full lines correspond to computed values and experimental points to the values obtained at heating rates of $5,10,20,40$, and $80 \mathrm{~K} \cdot \mathrm{min}^{-1}$ (for the T-HR-T diagram) and temperatures of 457,460 , and $463 \mathrm{~K}$ (for the T-T-T diagram). That is, the computed T-HR-T curves fit the experimental points when $\beta_{o}=20 \mathrm{~K} \cdot \mathrm{min}^{-1}$, as clearly shown in the inset of Fig. 12. However, in general, the agreement between experimental and computed values for different heating rates is quite good in the T-HR-T curves, and most significantly, when comparing both the T-T-T and the T-HR-T diagrams, regardless of the fact that the calculated T-T-T curves were obtained from continuous heating data. The agreement in temperature lies between $\pm 5 \mathrm{~K}$ in the experimentally measured range

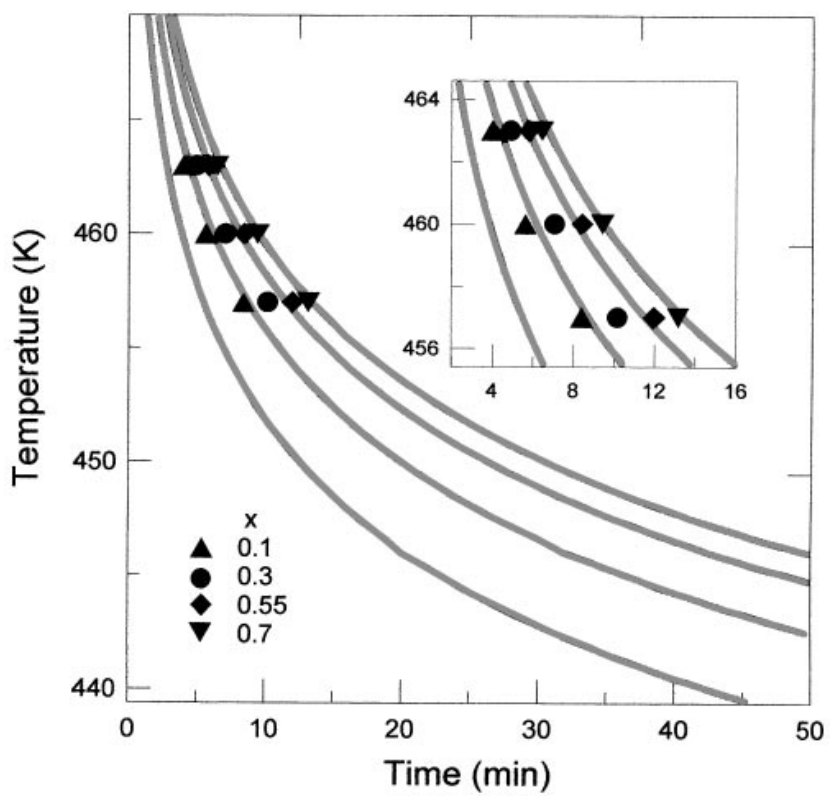

FIG. 11. T-T-T diagram of primary crystallization of Te from the amorphous $\mathrm{Ga}_{20} \mathrm{Te}_{80}$ alloy. Experimental points: $(\boldsymbol{\Delta}) x=0.1 ;(0) x=$ $0.3 ;(\diamond) x=0.55$; and $(\boldsymbol{\nabla}) x=0.7$. Continuous curves: calculated values.

but the predicted values are expected to have larger error bars when extended in regions not covered by experiment because the description in terms of activation energy is rather simplified with respect to reality.

\section{CONCLUSIONS}

A new procedure has been developed to obtain accurate data for the rate of phase transformation from continuous heating DSC measurements by subtraction of the heat capacity change contribution to the total calorimetric signal.

The effect of both base line instability and uncertainty on the exact position of the onset and end of the transformation exotherm in the isothermal data obtained by current DSC equipment on the determination of the rate of crystallization has been evaluated.

The link between the error in the evaluation of both the apparent activation energy and the pre-exponential factor of the rate constant from calorimetric data has been discussed. Very similar values for the apparent activation energy are shown to be expected, independently of the particular method used to process the experimental data.

The construction of predictive transformation diagrams, T-T-T and T-HR-T, which may extend the transformation temperatures to ranges wider than those measured experimentally by DSC by use of empirical crystallization kinetic parameters is discussed.

The overall procedure has been applied to study the crystallization kinetics of an amorphous alloy, $\mathrm{Ga}_{20} \mathrm{Te}_{80}$, 


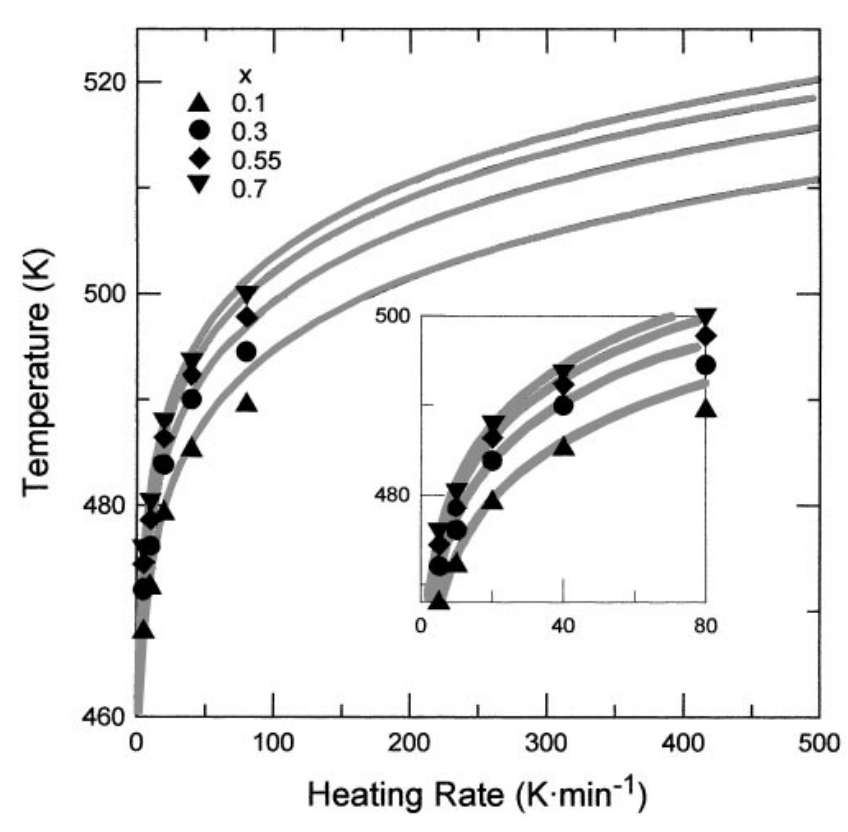

FIG. 12. T-HR-T diagram of primary crystallization of Te from the amorphous $\mathrm{Ga}_{20} \mathrm{Te}_{80}$ alloy. Experimental points: $(\mathbf{\Lambda}) x=0.1 ;(\boldsymbol{O}) x=$ 0.3 ; $\diamond) x=0.55$; and $(\nabla) x=0.7$. Continuous curves: calculated values.

for which the primary crystallization of hexagonal Te is accompanied by a significant heat capacity change of the sample. The kinetic parameters obtained from the primary crystallization study have been transformed and are presented in the form of the low temperature part of both the T-T-T and the T-HR-T transformation diagrams.

\section{ACKNOWLEDGMENTS}

This work has been supported by CIRIT (project no. 1995SGR-00514) and CICYT (project nos. MAT960692 and MAT96-0769) which are acknowledged. One of the authors (M. Fontana) gratefully acknowledges the grant released by the Instituto de Cooperación Iberoamericana of Spain. Thanks are also due to Dr. B. Arcondo and Professor H. Sirkin for the preparation of the amorphous alloys and to Dr. J. Zhu for her help in calorimetric measurements.

\section{REFERENCES}

1. H. J. Borchardt and F. Daniels, J. Am. Chem. Soc. 79, 41 (1957).

2. E. S. Freeman and B. Carroll, J. Phys. Chem. 62, 394 (1958).

3. A. W. Coats and J.P. Redfern, Nature 201, 64 (1964).

4. P. Hugo, S. Wagner, and T. Gnewikiw Thermochim. Acta 225, 143 (1993).

5. M. T. Clavaguera-Mora, M. D. Baró, S. Suriñach, and N. Clavaguera, J. Mater. Res. 5, 1201 (1990).

6. S. Suriñach, M. D. Baró, J. A. Diego, N. Clavaguera, and M. T. Clavaguera-Mora, Acta Metall. Mater. 40, 37 (1992).

7. M. T. Clavaguera-Mora, S. Suriñach, M. D. Barò, and N. Clavaguera, J. Phys. D: Appl. Phys. 25, 803 (1992).

8. N. Clavaguera, J. Non-Cryst. Solids, 162, 40 (1993).
9. N. Clavaguera and M. T. Clavaguera-Mora, Mater. Sci. Eng. A179/180, 288 (1994).

10. C. S. Oh and D. N. Lee, Calphad 16, 317 (1992).

11. D. Mouani, G. Morgant, and B. Legendre, J. Alloys Comp. 226, 222 (1995).

12. H. E. Kissinger, Anal. Chem. 29, 1702 (1957).

13. T. Ozawa, Bull. Chem. Soc. Jpn. 38, 1881 (1965).

14. G. Fontana, H. Sirkin, M. Rosen, D. Kurlat, and E. Frank, Rev. Brasileira de Fisica 9, 1 (1979).

15. M. G. Fontana and B. Arcondo, J. Mater. Sci. 30, 734 (1995).

16. M. Fontana, Master Thesis, Universitat Autónoma de Barcelona, 1995.

17. U. Köster and U. Herold, in Glassy Metals I, edited by H.J. Güntherodt and H. Beck, Topics Appl. Phys. (Springer, Berlin, 1981), Vol. 46, p. 225.

18. R.F. Speyer, Thermal Analysis of Materials, (Marcel Dekker, 1994).

19. R. F. Speyer, J. Mater. Res. 8, 675 (1993).

20. J. W. Christian, Theory of Transformations in Metals and Alloys, 2nd ed. (Pergamon, Oxford, 1975).

21. D. W. Henderson, J. Non-Cryst. Solids 30, 301 (1979).

22. H. S. Chen, J. Non-Cryst. Solids 27, 257 (1978).

\section{APPENDIX}

The initial stages of the crystallization process can be visualized as the formation of nuclei which, on growing, give rise to a concentration profile ahead of the interface, schematically shown in Fig. A1. Under the hypothesis of attainment of metastable equilibrium at the interface, at this location the concentration of the remaining nontransformed phase stabilizes to the value $c^{*}$. The respective values of the concentration in the crystal and at the interface are established from the free energy diagram shown in Fig. A2. As crystallization proceeds, the mean composition of the remaining phase evolves from $c_{o}$ to $c^{*}$. Then, the metastable heterogeneous equilibrium is reached all over the sample. During the transformation, the volume fraction occupied

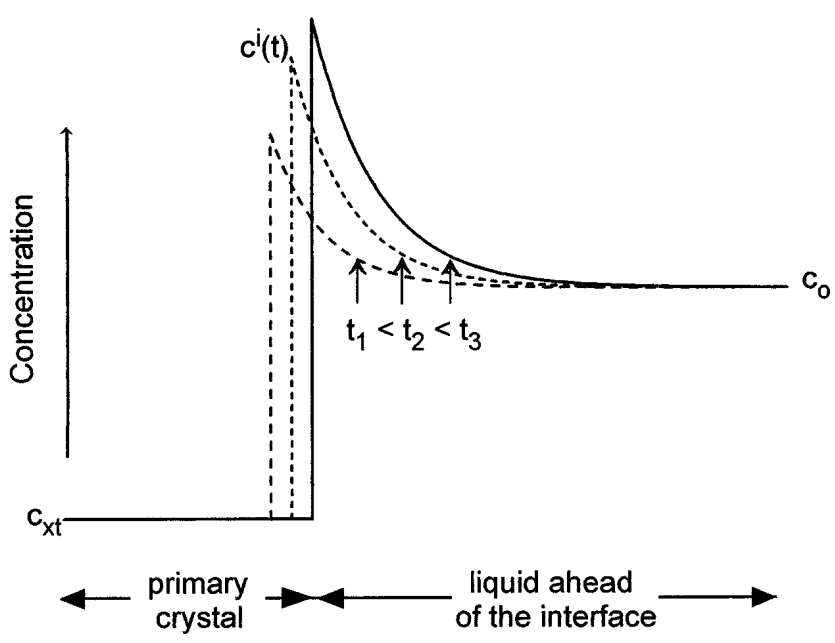

FIG. A1. Schematic view of the establishment of a concentration profile in the disordered phase ahead of the interface of a growing grain. 


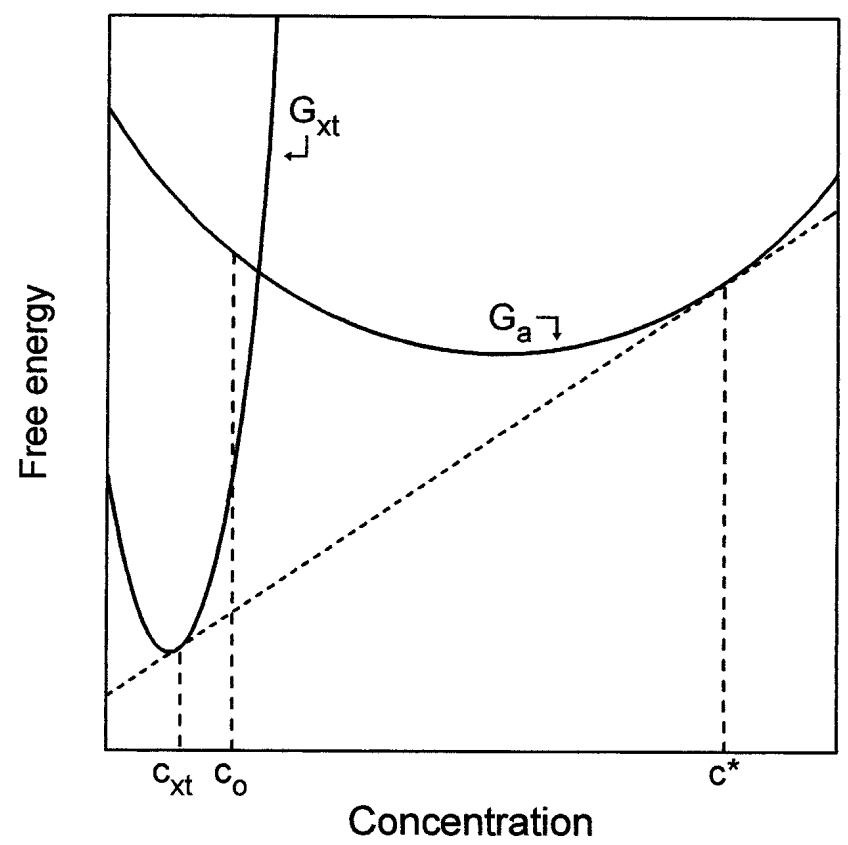

FIG. A2. Free energy curves of the crystalline and disordered solution phases at temperature far below stable equilibrium between them.

by the primary crystals is $x f_{1}$, where $f_{1}$ is given by the lever rule,

$$
f_{1}=\left(c^{*}-c_{o}\right) /\left(c^{*}-c_{x t}\right) .
$$

Neglecting differences of density among the various coexisting phases, $f_{1}$ is also the mass fraction transformed at the end of the crystallization process. The heat flux evolving from the sample is

$$
\dot{Q}=\Delta \dot{H},
$$

with

$$
\begin{aligned}
\Delta H= & \left\{x \cdot f_{1} \cdot H_{x t}\left(c_{x t}, T\right)+\left(1-x \cdot f_{1}\right) \cdot H_{a}(c, T)\right\} \\
& -H_{a}\left(c_{o}, T\right),
\end{aligned}
$$

$$
\begin{aligned}
\frac{d\left\{x \cdot f_{1} \cdot H_{x t}\left(c_{x t}, T\right)\right\}}{d t}= & \dot{x} \cdot f_{1} \cdot H_{x t}+x \cdot f_{1} \cdot C_{p, x t} \cdot \beta, \\
\frac{d\left\{\left(1-x \cdot f_{1}\right) \cdot H_{a}\right\}}{d t}= & -\dot{x} \cdot f_{1} \cdot H_{a}+\left(1-x \cdot f_{1}\right) \\
& \cdot\left[\frac{\partial H_{a}}{\partial c} \cdot \dot{c}+C_{p, a} \cdot \beta\right],
\end{aligned}
$$

and

$$
\frac{d\left\{H_{a}\left(c_{o}, T\right)\right\}}{d t}=C_{p, a}\left(c_{o}, T\right) \cdot \beta .
$$

In these equations $H_{\varphi}$ and $\Delta C_{p, \varphi}$ stand for the enthalpy and heat capacity per unit mass, respectively, of phase $\varphi$ with $\varphi=x t$ for the primary crystals and $\varphi=a$ for the undercooled liquid. Further, it has been assumed that the composition of the crystal, $c_{x t}$, remains constant all over the transformation.

Provided that (i) $\left(\partial H_{a} / \partial c\right) \cdot \dot{c}$ is negligible and (ii) $H_{a}(c) \approx H_{a}\left(c^{*}\right) \approx H_{a}\left(c_{o}\right)$ and $C_{p, a}(c) \approx C_{p, a}\left(c^{*}\right) \approx$ $C_{p, a}\left(c_{o}\right)$ in the range $c_{o} \leqslant c \leqslant c^{*}$ the heat flux evolved from the sample may be divided in two terms, namely, the rate of enthalpy change of the reaction alone,

$$
\dot{Q}_{\text {reaction alone }}=\dot{x} \cdot f_{1} \cdot\left(H_{a}-H_{x t}\right),
$$

and the rate of sample heat capacity change from that of the undercooled liquid to that of the crystallization product,

$$
\dot{Q}_{\Delta C_{p}}=x \cdot f_{1} \cdot\left(H_{a}-H_{x t}\right) .
$$

In polymorphic and eutectic crystallization, Eqs. (A7) and (A8) with $f_{1}=1$ are exact, since the composition of the coexisting phases remains constant during the transformation. In primary crystallization, they are good approximate expressions of the mean instantaneous heat flux generated in the reaction. 